\title{
The Effects of Interpolation and Smoothing of Color Matching Functions
}

\author{
H. J. Trussell \\ Dept. of Electrical and Computer Engineering \\ North Carolina State University \\ Raleigh, NC 27695-7911
}

\author{
M. J. Vrhel \\ Color Savvy Systems Ltd. \\ 305 South Main Street \\ Springboro, $\mathrm{OH} 45066$
}

\section{Introduction}

The CIE color matching functions, which are the foundation of computational color, are constructed and tabulated from experimental data by using various interpolations and smoothing. This work shows how the exact form of the mathematical operations used to construct the tabulated values can produce a bias in the computation of tristimulus values when used with sampled data obtained from various measuring instruments. The practical limitations caused by the bias are discussed.

\section{Ideal Sampling and Interpolation}

To design or test color devices computer simulations are performed. The accuracy of the simulations in modeling analog devices depends upon correct sampling and processing of the sampled data. Unfortunately, color signals are not perfectly bandlimited. This means that any sampling will result in some error. The statistics of the error will depend upon the relationship between the sampling and reconstruction (interpolation) methods and the statistical characterization of the color signals.

The exact method used to construct the CIE color matching functions is not published. However, it is sufficient to demonstrate the effect of sampling and interpolation for a general case. The general sampling operation can be described by

$$
r_{s}(n)=\int_{-\infty}^{\infty} r_{a}(t) s_{n}(t) d t
$$

where $r_{a}(t)$ represents the analog signal in the continuous domain and $s_{n}(t)$ represents an arbitrary sampling function or aperture. Classical sampling is described by $s_{n}(x)=\delta(x-n)$.

Let $\mathbf{r}$ be a signal defined in an $\mathrm{N}$-dimensional space, $\Omega_{N}$, where vectors are defined as column vectors. The above sampling can be considered a subsampling of $\Omega_{N}$. The integral in the continuous case can be considered an inner product operator. The general sampling can be represented by

$$
r_{s}(n)=\mathbf{r}^{T} \mathbf{s}_{n}=<\mathbf{r}, \mathbf{s}_{n}>
$$

where $\mathbf{s}_{n}$ is the $n^{\text {th }}$ sampling vector. The resulting samples are combined into a vector which corresponds to a sampled signal

$$
\mathbf{r}_{s}=\left[r_{s}(1), r_{s}(2), \cdots, r_{s}(M)\right]^{T}
$$

The sampling reduces the dimensionality of the space from $\mathrm{N}$ to $\mathrm{M}$. The sampled signal can be represented by a linear operation

$$
\mathbf{r}_{s}=\mathbf{S}^{T} \mathbf{r}
$$

where $\mathbf{S}$ is an $N \times M$ matrix and $\mathbf{r}_{s}$ is a $M \times 1$ vector. The vector $\mathbf{r}_{s}$ which contains the sample values represents a vector in the $M$ dimensional subspace of $\Omega_{N}$ spanned by the columns of $\mathbf{S}$. This vector is defined by the projection

$$
\mathbf{r}_{p}=\mathbf{S}\left(\mathbf{S}^{T} \mathbf{S}\right)^{-1} \mathbf{S}^{T} \mathbf{r}
$$

From this representation, it is clear that the sampling process can be thought of as an orthogonal projection operator from a vector space, $\Omega_{N}$, to a subspace, $\Omega_{S} \subset \Omega_{N}$.

A reconstruction (interpolation) method is a mapping from a lower dimensional space defined by samples to a higher dimensional space. For example, using eq.(??)

$$
\mathbf{r}_{i}=\mathbf{H}_{M} \mathbf{r}_{s}=\mathbf{H}_{M} \mathbf{S}^{T} \mathbf{r}
$$

the interpolation matrix, $\mathbf{H}_{M}$ is $N \times M$. Because of the projection interpretation of sampling, interpolation can also be represented as a mapping from $\Omega_{S}$ to a subspace of $\Omega_{N}$. If we continue to use linear mappings, then the reconstruction can be written

$$
\hat{\mathbf{r}}=\mathbf{H} \mathbf{r}_{p}=\mathbf{H S}\left(\mathbf{S}^{T} \mathbf{S}\right)^{-1} \mathbf{S}^{T} \mathbf{r}
$$


where the caret denotes an estimate. The interpolation lies in a subspace defined by the columns of the $N \times N$ matrix $\mathbf{H}, \hat{\mathbf{r}} \in \Omega_{H}$. Note that $\mathbf{H}$ and $\mathbf{H}_{M}$ represent the same mapping but in different representations of the subspace. For ease of notation and to avoid confusion, all matrices in the remainder of the paper will be $N \times N$.

It is common to choose interpolation operators which can exactly interpolate themselves, which means that $\mathbf{H H}^{T}=\mathbf{H}$. If $\mathbf{H}$ is symmetric as is usually the case, this implies that $\mathbf{H}$ is a projection operator. However, the projection need not be orthogonal. The reconstruction is exact if $\mathbf{r} \in \Omega_{H}=\Omega_{S}$. In this case, $\mathbf{P}=\mathbf{H S}\left(\mathbf{S}^{T} \mathbf{S}\right)^{-1} \mathbf{S}^{T}$ is the identity operator on $\Omega_{H}$.

Errors can arise in several ways. One is that the sampling and interpolation are not matched, i.e. $\Omega_{H} \neq \Omega_{S}$. A second is that $\mathbf{r}$ is not completely contained within $\Omega_{H}$. A combination of the two is the most likely case. The fraction of the signal power of $\mathbf{r}$ in $\Omega_{H}^{c}$ is a measure of the error where $\Omega_{N}=\Omega_{H}^{c} \times \Omega_{H}$.

\section{Problem Formulation}

For the computation of CIE tristimulus values from measured data, the CIE recommends interpolating the signal to the resolution of their tabulated color matching functions, at one $\mathrm{nm}$ intervals from $360 \mathrm{~nm}$ to 830 $\mathrm{nm}$, multiplying the functions and summing. The recommended interpolation is convolution with a Laplacian function denoted by $\mathbf{H}_{L}[?]$.

The CIE color matching functions, $\mathbf{A}=$ $\left[\mathbf{a}_{1}, \mathbf{a}_{2}, \mathbf{a}_{3}\right] \in \Omega_{N}$ represent a standard observer. Denote the CIE sampling operator by $\mathbf{S}_{C}$. It is of interest whether the composite operator

$$
\mathbf{P}_{C}=\mathbf{H}_{L} \mathbf{S}_{C}
$$

is the identity operation on the proper subspace. The fact that most instruments produce data at equal wavelength intervals leads one to assume that the subspace of interest is the space defined by the subsampling operator, $\mathbf{S}_{K}$ where the vectors of this matrix are defined by $N / K$ vectors with one in the $n K$ position:

$$
\mathbf{s}_{n}=[0 \cdots 010 \cdots 0]
$$

The limitation of the range of most instruments to smaller domains than $360-830 \mathrm{~nm}$ can easily be represented with the above mathematics. The extrapolation problem can be addressed separately from the interpolation problem but the method is similar [?].

The actual tristimulus values are obtained by taking the inner products in the original vector space

$$
\mathbf{t}=\mathbf{A}^{T} \mathbf{r}
$$

The computation of the tristimulus values from sampled data is given by

$$
\mathbf{t}_{C}=\mathbf{A}_{C}^{T} \mathbf{H}_{L} \mathbf{S}_{K} \mathbf{r}
$$

where the color matching functions which make up the matrix, $\mathbf{A}_{C}$, are themselves obtained by a sampling and interpolation process

$$
\mathbf{A}_{C}=\mathbf{H}_{C} \mathbf{S}_{C} \mathbf{A}
$$

where $\mathbf{H}_{C}$ represents the interpolation used on the sampled color matching function data.

When the tristimulus values are computed from sampled data, the ideal interpolation method would minimize the function:

$$
\Phi\left(\mathbf{H}_{i}\right)=E\left\{\left\|\mathbf{A}^{T} \mathbf{r}-\mathbf{A}^{T} \mathbf{H}_{i} \mathbf{S}_{K} \mathbf{r}\right\|^{2}\right\}
$$

where the expected value operator, $E\{\cdot\}$, is taken over all possible reflectances $\mathbf{r} \in \Omega_{N}$. The optimal interpolation is defined by the solution to the equation

$$
\mathbf{A} \mathbf{A}^{T} \mathbf{K}_{r r} \mathbf{S}_{K}=\mathbf{A} \mathbf{A}^{T} \mathbf{H}_{i} \mathbf{S}_{K} \mathbf{K}_{r r} \mathbf{S}_{K}^{T}
$$

where $\mathbf{K}_{r r}=E\left\{\mathbf{r r}^{T}\right\}$. Note that $\mathbf{H}_{i}$ is an $N \times N$ matrix but has fewer than $2 N$ degrees of freedom since it is Toeplitz.

Since the ideal color matching functions are used in this formulation, any interpolation that reproduces $\mathbf{r}$ will be optimal. This in turn would require that $\mathbf{r}$ be contained in the subspace defined by $\mathbf{H}_{i}$ and $\mathbf{S}_{K}$. Alternatively, an optimal solution can be obtained if the interpolation can reproduce the projection of $\mathbf{r}$ onto the 3-D subspace defined by $\mathbf{A}$.

For practical comparison we use the tabulated CIE color matching functions which are represented by eq.(??). The problem of finding the best interpolating function is now to minimize

$$
\Phi(\mathbf{H})=E\left\{\left\|\mathbf{A}_{C}^{T} \mathbf{r}-\mathbf{A}_{C}^{T} \mathbf{H S}_{K} \mathbf{r}\right\|^{2}\right.
$$

The optimal $\mathbf{H}$ is the solution of

$$
\begin{aligned}
& \mathbf{H}_{C} \mathbf{S}_{C} \mathbf{A} \mathbf{A}^{T} \mathbf{S}_{C}^{T} \mathbf{H}_{C}^{T} \mathbf{K}_{r r} \mathbf{S}_{K}^{T}= \\
& \mathbf{H}_{C} \mathbf{S}_{C} \mathbf{A} \mathbf{A}^{T} \mathbf{S}_{K}^{T} \mathbf{H}_{C}^{T} \mathbf{H} \mathbf{S}_{K} \mathbf{K}_{r r} \mathbf{S}_{K}^{T}
\end{aligned}
$$

which is obtained using the generalized inverse.

At this stage, it can be seen that the optimal solution is a combination of sampling function, the CIE interpolation function and the statistical characterization of the ensemble. The effect of the sampling choice and interpolation functions depends on the $\mathbf{K}_{r r}$. For our cases, it will be assumed that the reflectance signals will be nearly bandlmited as was illustrated in [?]. 
The color matching functions of $\mathbf{A}$ will have the same property.

The matrix $\mathbf{H}_{C}^{T} \mathbf{H}$ is an interpolation of the CIE interpolating functions. As was done previously, assume that the interpolating function is created so that it interpolates itself exactly (sinc functions do this). Further assume that $\mathbf{K}_{r r}=\sigma^{2} \mathbf{I}$. Then, it can be shown that $\mathbf{H}=\mathbf{H}_{C}$ is a solution. The implication is that for the case of maximum ignorance about the ensemble of reflectances $\left(\mathbf{K}_{r r}=\sigma^{2} \mathbf{I}\right)$, no interpolation method is better than that used for the tabulated data.

\section{Results}

To simulate the nearly bandlimited assumption of the color matching functions and the reflectances, random bandlimited signals were generated, then noise in the complement space was added at various power levels. The simulation reflects what could be done in a practical test. The true CMFs are actually unknown. What is known is the tabulated CIE CMFs. The reflectances can be known exactly since they can be measured with high precision spectrophotometers. Thus, a typical simulation as done in [?] used the tabulated CMFs and the measured reflectances to obtain the "standard" against which interpolation methods were compared. The results of [?] show that it is virtually impossible to produce a smaller tristimulus (XYZ or Lab) error than obtained with the CIE recommended interpolation. This result is duplicated in the simulations.

The color matching functions are very smooth and should be well approximated by bandlimited functions. Thus, the most appropriate case is for the noise added to the random bandlimited signals to be small. On the other hand, the reflectances have a more varied degree of smoothness and are modeled by a higher noise power added to the bandlimited signal. This modeling reflects the data presented in [?] and [?]. The color matching functions and the reflectances were both generated using the mathematical form

$$
\mathbf{v}=\mathbf{P}_{d} \mathbf{x}+\sigma_{k} \mathbf{P}_{d c} \mathbf{x}
$$

where $\mathbf{P}_{d}$ represents the projection operator onto the bandlimited space, $\mathbf{P}_{d c}$ represents the projection operator onto the complement of the bandlimited space, vector $\mathbf{v}$ is the generated vector, and $\mathbf{x}$ is a normal, zero mean, unit variance random vector. The parameter $\sigma_{k}$ determines the power of the signal outside the bandlimited space. The generated vectors were sampled and interpolated with either a windowed (Hanning) sinc function, or the CIE recommended interpolator (Laplacian). The inner product of the interpolated color matching functions with the interpolated reflectance spectra were computed, and compared in terms of mean square error with either the ideal inner product or with other interpolation methods.

The results of the simulation are shown in Table 1. The first two columns indicate the out of band portion $\left(\sigma_{k}\right)$ used in generating the simulated signals where $\sigma_{a}$ is for the color matching functions, and $\sigma_{r}$ is for the reflectance spectra. Columns 3 and 4 compare the ideal inner product (that is using the non-interpolated functions) with the result of using the CIE and DSP (windowed sinc) interpolations of the reflectance samples respectively, when the CIE interpolated CMF is used to compute the tristimulus values. In all cases, the DSP interpolation is better. In practice, the noninterpolated CMFs are unavailable and the CIE provides interpolated CMFs. Columns 5 and 6 indicate the result when the ideal CMFs are replaced with the CIE interpolated functions. Column 5 uses the DSP interpolation for reflectance spectra and column 6 uses the CIE interpolation. Comparing 5 and 6 simulates results obtained by previously presented experiments. For this case, the CIE interpolation of the reflectance spectra is better than the DSP interpolation.

\section{Conclusion}

The results presented explain results in previous work testing interpolation methods. The fact that the classical DSP interpolation methods produce better estimates of the original reflectance vectors but poorer estimates of the tristimulus vectors is a result of the interpolation method used to produce the tabulated CIE CMFs. It is noted that the CIE CMFs provide a very good standard and allow communication of the color data. However, they should not be considered as giving the most accurate estimates of the tristimulus values which characterize a human observer.

\section{References}

[1] C. L. Grard and H. J. Trussell, "Interpolation and Extrapolation for Accurate Color Measurement," Proc. IEEE Int. Conf. on Image Proc., Vol. II, pp350-355, 23-26 Oct. 1995, Washington, DC.

[2] Colorimetry; 2nd Ed., CIE Publication 15.2, Central Bureau of the CIE, Paris, 1986.

[3] H. J. Trussell and M. S. Kulkarni, "Sampling and Processing of Color Signals," IEEE Trans. Image Proc., Vol. 5, No. 4, pp. 677-681, April 1996.

[4] M. J. Vrhel, R. Gershon, and L. Iwan, The Measurement and Analysis of Object Reflectance Spectra, Color Research and Application, vol. 19, no. 1, pp. 4-9, Feb 1994. 
Table 1: $L^{*} a^{*} b^{*}$ Errors for Interpolators

$\begin{array}{rrrrrr}\sigma_{a} & \sigma_{r} & \text { cie/ideal } & \text { dsp/ideal } & \text { cie/dsp } & \text { cie/cie } \\ 0.0500 & 0.0500 & 0.3427 & 0.3319 & 0.1092 & 0.0463 \\ 0.2000 & 0.0500 & 0.4652 & 0.2830 & 0.4040 & 0.1652 \\ 0.5000 & 0.0500 & 0.7937 & 0.3124 & 0.8137 & 0.3360 \\ 0.0500 & 0.2000 & 0.3059 & 0.2994 & 0.1001 & 0.0632 \\ 0.2000 & 0.2000 & 0.4631 & 0.3097 & 0.3726 & 0.1822 \\ 0.5000 & 0.2000 & 0.9219 & 0.4656 & 0.8789 & 0.4300 \\ 0.0500 & 0.5000 & 0.2978 & 0.2866 & 0.1126 & 0.0803 \\ 0.2000 & 0.5000 & 0.5508 & 0.3977 & 0.3979 & 0.2432 \\ 0.5000 & 0.5000 & 0.9712 & 0.6722 & 0.8628 & 0.5678\end{array}$

